\title{
Risk Factor Assessment in Stroke - Prospective Study in GGH Nalgonda
}

\author{
Author \\ Dr C. Yadavendra Reddy MD General Medicine \\ Assistant Professor in Medicine, Government General Hospital Nalgonda, India \\ Email: chamareddy_07@yahoo.com
}

\begin{abstract}
Background: Stroke is the second most common cause of death and major cause of disability worldwide. The objective of this study is to assess the risk factors in stroke for the patients in GGH Nalgonda.

Methods: A Prospective study was conducted on patients in District Hospital Nalgonda from Dec 2018 to April 2019. All the patients admitted with stroke were assessed of age, sex in the hospital.

Result: A total of 100 patients with stroke were included in the study. Of the 100 patients 80 had ischaemic stroke and 20 patients had haemorrhagic stroke. The stroke was predominant in males with $64 \%$ and females with 36\%.The mean age of this incidence was 50. It was observed that predisposing factor for stroke was age in 72 patients; HTN in 65 patients; DM in 45 patients; Smoking in 40 patients; Alcohol in 50 patients; Hypercholestrolemia in 35 patients; while others contributed in 30 patients.

Conclusion: This study revealed that senility was predisposing factor in stroke accompanied with lack of awareness of comorbid condition like HTN,DM leading to stroke.
\end{abstract}

\section{Introduction}

Stroke is the most common, life threatening disease major cause of morbidity and mortality worldwide especially in South Asian Subcontinent ${ }^{[1,2]}$. Strokeis affecting millions of people in both developed and developing Countries ${ }^{[3]}$. Stroke is the First leading cause of death and the most prominent factor related to disability adjusted life years with high social economic $\operatorname{cost}^{[4]}$. In addition stroke has a high recurrence rate among non-fatal cases due to extremely low treatment rates and poor adherence to essential treatment of secondary prevention ${ }^{[5,6]}$. According to survey conducted by Indian council of Research in 2006, it was found that prevalence of stroke varies from 40 to 270 in 1,00,000 population in different regions of India and most of population effected are $<40$ years age ${ }^{[7]}$. According to WHO stroke is a rapidly developed clinical sign of focal disturbance of cerebral function of presumed vascular origin and of more than $24 \mathrm{hr}^{[8]}$.

The risk factor for stroke may be non-modifiable (Race, Age, Sex, LBW) Modifiable (HTN, Dyslipidaemia, DM, Tobacco, AF, Cardiac Disorder, Sickle Cell disorder, Diet \& BMI) and potential risk factor (Metabolic syndrome, Alcohol, Drug Abuse, Sleep apnea, OC pills other factor include low education, poor economic condition, tobacco, infection, BMI (obesity, BMI>30), $\operatorname{Diet}^{[9]}$.

\section{Materials \& Methods}

Observational study was conducted in GGH Nalgonda from Dec 2018 to April 2019. During this period all the patients admitted with CVA were assessed. The patients were asked about their habits and the presence of any comorbid factors leading to stroke. The risk factor identified included - Old age, Hypertension, DM, Alcoholic, Smoking. The most 
important observation was the irregular use of drugs for these comorbid conditions leading to stroke. Further consumption of alcohol was another important factor leading to stroke.

\section{Observation \& Results}

A total of 100 patients were enrolled during the study period. The mean age of the patients was 50 . The incidence of the stroke was predominant in Males (64\%) and Females (36\%) and most of the patients above age 40 years. Out of the patients A/W stroke $80 \%$ had Ischaemic stroke and $20 \%$ had Haemorrhagic stroke. Out of the patients with stroke (combined) $70 \%$ had HTN; 75\% were age above 55 years; Alcoholics were 55\%; Diabetes were 50\%.
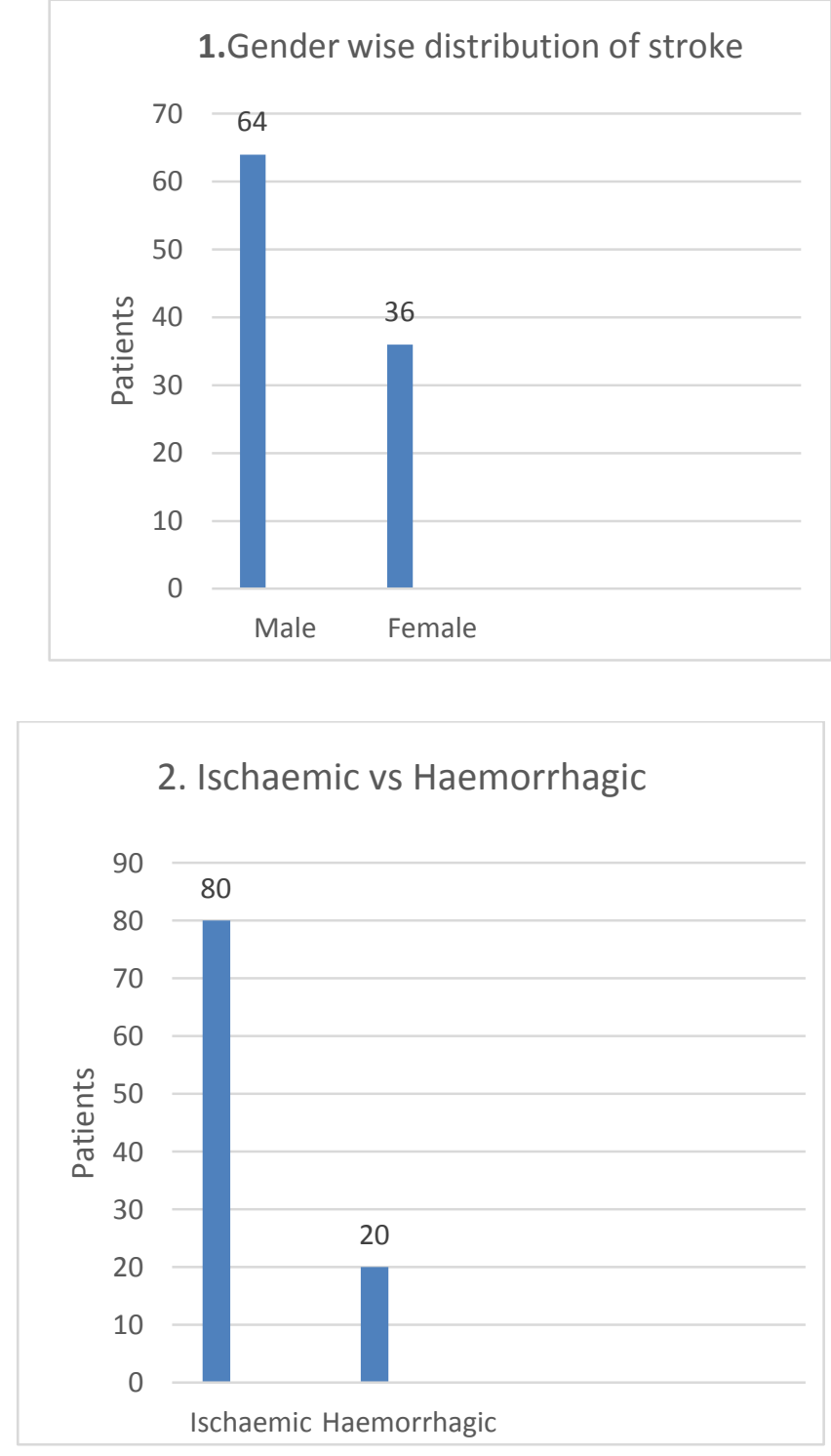

\section{Distribution of risk factors in stroke}

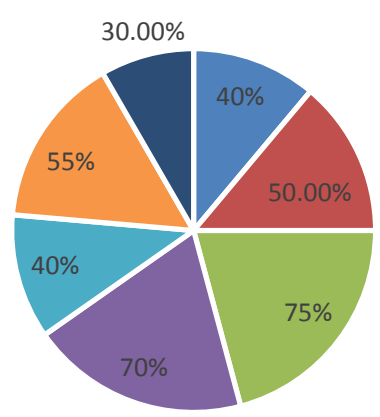

$$
\begin{array}{ll}
\square \text { Smoking } & \square \text { DM } \\
- \text { Age }>50 & \square \text { HTN } \\
\text { - Hypercholestermia } & \text { Alcohol } \\
\text { - Others } &
\end{array}
$$

\section{Discussion}

Stroke is an emergency which needs immediate hospitalization to treat \& save lives. A silent stroke does not have any outward symptoms \& cause damage to the brain and places the patient at increased risk for both TIA and major stroke in future $^{[10]}$.In the study it was observed the senility was a major contribution to stroke with comorbidities like HTN, DM, Aggravated the risk for stroke ${ }^{[11,12]}$. The risk of stroke is increased by Smoking, DM, Heart disease, TIA an Atrial fibrillations. The result were consistent with other publisher studies ${ }^{[13,14,15]}$. In our study Alcohol and Smoking were documented risk factors for stroke which increased the incidence of stroke ${ }^{[16]}$. The risk of stroke increased in HTN patients who were Heavy drinkers ${ }^{[17]}$. Further the increased risk of stroke in patient was due to lack of awareness of the risk factor like HTN, Alcohol leading to stroke. Therefore a few programs regarding stroke such a Audio visual programs printed information\& stroke service are to be introduced to create awareness.

\section{Conclusion}

The study revealed that Age, Gender, HTN, Diabetes, Smoking \& Dyslipidemia are the most common risk factor for stroke. Regular moderate degree of physical activity and tight control of HTN dyslipidemia \& Dmprevents substantial proportion 
of stroke incidence. A fundamental principle in disease prevention is that a large number of people exposed to a relatively low risk are likely to produce more cases than a small number of people exposed to high risk. Promoting healthy lifestyle and awareness regarding side effects of Smoking, Alcohol, should decreased the occurrence of stroke.

\section{References}

1. Cher, Kim Js-lifestyle factors and risk of stroke in seuolsouthkoera $\mathrm{J}$ stroke CV disease -1998

2. Das Sk-WHO steps stroke surveillance system feasibility in india. Indian $\mathbf{J}$ med res2009;

3. Mozaffarian. D et al. Heart disease and stroke statistic-2016-update- A Report from AHA

4. Yanetal. Rapaid health transition 1990-2010 findings for the Global Burden disease study 2010 lancet

5. Yusuf $\mathrm{S}$.etal. use of secondary prevention drugs for cardio vascular disease in high income and middle income and low income countries (Pure study) a prospective epidemic survey.

6. Cheny. $\mathrm{Y}$ et al use of drug treatment for secondary prevention of $\mathrm{CV}$ disease in urban and rural communities of china INT $\mathrm{J}$ of cardiology.

7. New delhi 2006 work shop report on stroke surveillance in India held on 13-15 Nov

8. WHO MONICA (Monitoring trends and determinants in $\mathrm{CV}$ disease) a major interim collaboration.

9. Fagan.SE David $\mathrm{CH}$ stroke - Pharmacotherapy pathological Approach.

10. Fagan.SE David CH stroke - Pharmacotherapy pathological Approach.

11. Idris, Thomson GA Sharama JC DM \& stroke.

12. Castilio, Kumral E Henriques stroke subtypes and Hypertension primary haemorrhagevs Infarction; Large vs Small artery disease.
13. Shinton $R$ Beevers $G$ met analysis relation between cigaratte smoking and stroke.

14. Kawachi, Coldith-Smoking cessation and decreased risk of stroke in women JAMA.

15. Camrgola alcohol consumption and stroke the epidemiologic evidence of stroke.

16. Puddy alcohol, HTN \& CV diseaseImplication for management.

17. Lang. T, Nicaud-Dane improving HTN among ex alcohol drinkers. 